\title{
Numerical and physical modeling of heat transfer in the exhaust system of a piston engine in stationary conditions
}

\author{
Leonid Plotnikov ${ }^{*}$, Alexandr Nevolin, and Mariya Misnik \\ Ural Federal University named after the first President of Russia B.N. Yeltsin, Turbines and engines \\ Department, 620002 Ekaterinburg, Mira str., 19, Russian Federation
}

\begin{abstract}
Thermomechanical perfection of exhaust systems largely determines the efficiency of the engine boost system. The article presents the results of numerical simulation and experimental study of heat transfer of gas flows in profiled exhaust systems of ICE. The description of the numerical simulation technique, the experimental setup, the configurations of the hydraulic systems under investigation, the instrumentation and the experimental features are given in the article. On the basis of numerical simulation, it has been established that the use of profiled sections with a cross-section in the form of a square in the exhaust system of an ICE leads to a decrease in the heat transfer rate to $5 \%$. The use of profiled sections in the form of a triangle in the system under consideration causes a more significant decrease in heat transfer, which reaches $11 \%$. Experimental studies qualitatively confirm the results of simulation.
\end{abstract}

\section{Introduction}

Technical and economic indicators of piston internal combustion engines (ICE) largely depend on the thermal-mechanical perfection of the processes taking place in the exhaust system $[1,2]$. The flow structure in the channels and the hydraulic resistance of the exhaust system determine the gas-dynamic design perfection. This has a direct impact on the degree of cylinder purification from exhaust gases and, accordingly, on the main characteristics of the working process of piston ICE. Many different works are devoted to this topic. For example, an integrated approach to improving the exhaust system of an automobile engine is presented in Ref. [3]. The improvement of the methods of calculations and modeling of the intake and exhaust processes in the conditions of gas-dynamic unsteadiness is described in the article [4]. Similar results are presented in Ref. [5]. The authors developed a mathematical model of a three-cylinder engine that takes into account the features of the thermal-mechanical processes in gas-air paths and in the engine cylinder. It can be noted that there are other works aimed at creating mathematical models for calculating the exhaust process in piston ICEs [6,7]. In turn, the thermal-mechanical perfection of the exhaust system configuration determines the thermal stress of its parts and assemblies, as well as the amount of heat transfer that can be produced in the turbocharger turbine in the

* Corresponding author: leonplot@mail.ru 
case of a supercharged engine. The importance of studying the gas parameters in the exhaust system on the efficiency of the turbocharger and the engine is shown in [8-10]. Thus, obtaining information about the flow structure and heat transfer intensity in the exhaust system of piston ICE is an urgent task for the development of engine building.

This paper presents the results of numerical simulation and experimental study of the heat transfer of gas flows in profiled exhaust systems for an engine of 8.2/7.1 dimension.

\section{Modeling parameters and description of the experimental base}

The studies were carried out, both with the help of mathematical modeling, and experimentally on a laboratory setup.

The mathematical model based on the STAR-CCM + software was developed to study the heat exchange of the gas flow in the exhaust system. The configuration of the investigated exhaust system of the piston engine is shown in Fig. 1. The exhaust valve was in the uppermost (open) position during the simulation (the valve lift was $9 \mathrm{~mm}$ ). Studies were carried out for three configurations of the exhaust pipeline. In the first case, the exhaust pipeline had a constant circular cross-section along the entire length. In the second and third cases, the exhaust pipeline had a profiled section with cross sections in the form of a square and a triangle, respectively. The length of the profiled section was $250 \mathrm{~mm}$ with a total length of the exhaust pipe equal to $450 \mathrm{~mm}$. All the considered pipes had an equivalent hydraulic diameter of $30 \mathrm{~mm}$. The cylinder diameter was $82 \mathrm{~mm}$.

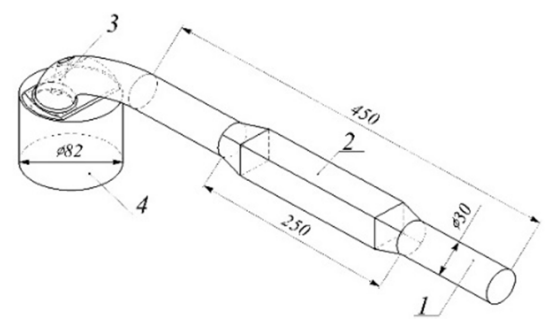

Fig. 1. Configuration of the exhaust system under study: 1 - exhaust pipe; 2 - profiled section; 3 - exhaust valve; 4 - cylinder

The boundary conditions in the simulation were as follows. The overpressure in the cylinder ranged from 90.15 to $130 \mathrm{kPa}$ at the model inlet. The pressure at the model output was $90 \mathrm{kPa}$. Thus, the pressure difference between the inlet and outlet was from 0.15 to $40 \mathrm{kPa}$. Under such conditions, the gas flow rate at the outlet of the exhaust pipeline was from 10 to $130 \mathrm{~m} / \mathrm{s}$. Dry air with a temperature of $120{ }^{\circ} \mathrm{C}$ was used as a working medium. The k-e model of turbulence was used to model turbulent flow. The problem was solved in a stationary formulation. The mesh of the exhaust system consisted of 627156 cells.

Experimental studies of heat transfer in exhaust systems were carried out for the configurations described above. The experiments were carried out under steady-state conditions in the air flow in the exhaust system. The piston engine exhaust valve was fixed in the uppermost position, and the air movement was created by a compressor that created an overpressure in the cylinder. The average airflow velocity in the exhaust pipeline varied from 10 to $90 \mathrm{~m} / \mathrm{s}$. Three control sections were chosen at distances $l_{x}$ from the window in the cylinder head of $100 \mathrm{~mm}, 200 \mathrm{~mm}$ and $300 \mathrm{~mm}$ in the pipeline under investigation. The internal surface of the exhaust systems had a technically smooth surface with an average roughness value of $6.3 \mu \mathrm{m}$. Two sensors of the thermo-anemometer were installed in each section to measure the flow velocity $w_{x}$ and the local heat transfer coefficient $\alpha_{x}$. In each control section, the measurement of the $\alpha_{x}$ was carried out in succession in 4 places separated by an angle of $90^{\circ}$ from each other. First, the values of $\alpha_{x}$ for each control section 
were averaged to obtain the heat transfer coefficient in the cross section. Then the values of $\alpha_{x}$ for all control sections were averaged to obtain the heat transfer coefficient of the exhaust system. The constant-temperature thermo-anemometer was used to determine the instantaneous values of the air flow velocity $w_{x}$ and the local heat transfer coefficient $\alpha_{x}$. In our case, a nichrome thread with a diameter of $5 \mu \mathrm{m}$ and a length of $5 \mathrm{~mm}$ was a sensitive element of the thermo-anemometer sensors. The method for determining $w_{x}$ and $\alpha_{x}$ is described in detail in Ref. [11].

It is known that cross-profiling of the pipeline causes changes in gas-dynamics in the hydraulic system [12], and this should lead to a change in the heat transfer intensity.

\section{Mathematical modeling of heat transfer in the exhaust system}

The influence of the pipeline cross-sectional shape on the heat transfer intensity in the exhaust system of a piston ICE was evaluated on the basis of numerical simulation (Fig. 2).

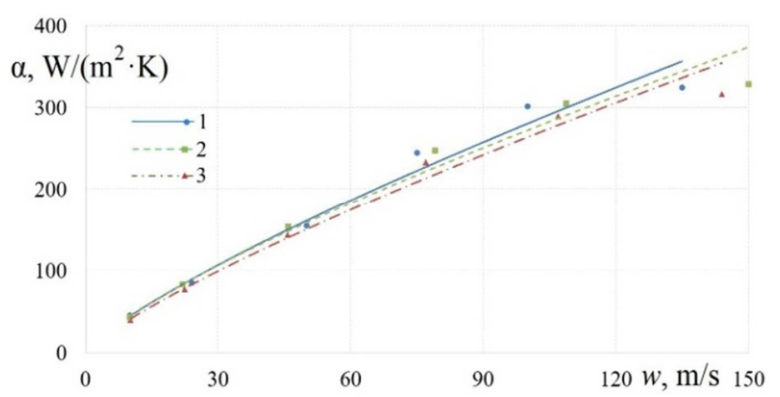

Fig. 2. Calculated dependences of the heat transfer coefficient in the exhaust pipeline on the air flow velocity for different shapes of the channel cross-section: 1 - circle; 2 - square; 3 - triangle

From Fig. 2 it can be seen that a decrease in the heat transfer intensity is observed when using an exhaust pipeline with a section with a square cross-section at all air flow rates. This reduction is up to $5 \%$ compared to the exhaust system of constant circular crosssection. At the same time, the greatest decrease in the heat transfer intensity occurs at high flow rates (more than $100 \mathrm{~m} / \mathrm{s}$ ). A similar effect was established for an exhaust system with a profiled section with a triangular cross section. A decrease in the heat transfer intensity is observed at all air flow velocities, which can reach $12 \%$ in comparison with the exhaust system of a constant circular cross section. Apparently, this phenomenon is associated with the presence of stable vortex structures formed in the corners of channels with a crosssection in the form of a square and a triangle, which prevent the formation of a stable thermal layer and, accordingly, reduce the heat exchange of the gas with the walls.

\section{Experimental data on heat transfer in the exhaust system}

Experimental studies have confirmed the results of numerical simulation at a qualitative level (Fig. 3). Differences in the numerical values may be due to the error in the experimental determination of the heat transfer coefficient and slight differences in the investigated configurations of the systems (in particular, in the surface roughness).

The decrease in heat transfer intensity is observed when using profiled sections in the exhaust system of the internal combustion engine at all flow rates (compared to the exhaust system of a constant circular cross section). Experiments have shown that a decrease in the intensity of heat transfer is observed up to $25 \%$ when using a section with a square crosssection in the exhaust system in comparison with the base system. Similar data were 
obtained using an exhaust pipeline with a triangular cross-section. In this case, the maximum reduction in the heat transfer coefficient reaches $33 \%$ in comparison with the exhaust system of a constant circular cross section.

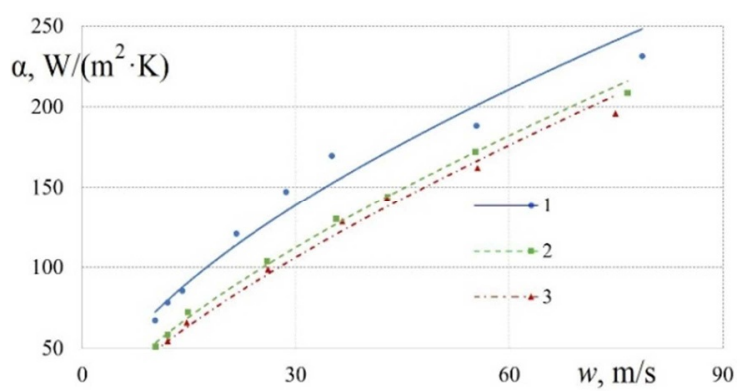

Fig. 3. Experimental dependences of the local heat transfer coefficient in the exhaust pipeline on the air flow velocity for different shapes of the channel cross-section: 1 - circle; 2 -square; 3 - triangle

It is noteworthy that a decrease in the $\alpha_{x}$ was also observed in the study of local heat transfer in the exhaust system under conditions of gas-dynamic nonstationarity (pulsating flows) at all crankshaft speeds $[13,14]$.

\section{Summary}

Thus, the following main conclusions can be made on the basis of the conducted research:

- mathematical model and experimental setup have been developed that simulate the physical and geometric configuration of the exhaust system of piston ICE;

- it has been established that the presence of a profiled section in the exhaust system leads to a decrease in the heat transfer intensity up to $30 \%$; this factor can positively affect the operation of the engine with supercharging, because in this case less heat will go into the walls of the pipeline in the exhaust process.

The work has been supported by the Russian Science Foundation (grant No. 18-79-10003).

\section{References}

1. J.B. Heywood, Internal combustion engine fundamentals, (McGraw-Hill, New York, 1988).

2. B.P. Zhilkin, V.V. Lashmanov, L.V. Plotnikov, Improvement of Processes in the GasAir Tracts of Piston Engines (Ural Publishers, Ekaterinburg, 2015).

3. E. Lazarev, V. Lazarev, A. Pomaz, WIT Trans. Ecol. Environ. 224(1), 91-99 (2017).

4. Y.A. Grishin, V.A. Zenkin, R.N. Khmelev, J. Eng. Phys. Therm. 90, 965-970 (2017).

5. H. Karabulut, H. Solmaz, D. Ipci, Appl. Therm. Eng. 121, 750-760 (2017).

6. Y. Liu, F. Zhang, Z. Zhao, T. Cui, Z. Zuo, S. Zhang, En. Proc. 142, 1172-1178 (2017).

7. H. Boden, SAE Int. J. Engines. 7(3), 1579-1584 (2014).

8. B. Franzke, S. Pischinger, P. Adomeit, C. Schernus, J. Scharf, T. Uhlmann, SAE Int. J. Materials Manufact. 9(2), 276-285 (2016).

9. L. Huang, C. Ma, Y. Li, J. Gao, M. Qi, Appl. Therm. Eng. 141, 1080-1091 (2018).

10. N. Zhu, L. Lü, D. Yang, Chinese Int. Comb. Engine Engin. 36(6), 8-13 (2015).

11. L.V. Plotnikov, B.P. Zhilkin, Int. J. Heat Mass Transfer. 115, 1182-1191 (2017).

12. S.S. Kutateladze, Heat transfer and flow resistance (Atomizdat, Moscow, 1990).

13. L.V. Plotnikov, B.P. Zhilkin, Y.M. Brodov, Proc. Eng, 206, 80-85 (2017).

14. Y.M. Brodov, B.P. Zhilkin, L.V. Plotnikov, Tech. Phys. 63(3), 319-324 (2018). 